\title{
ON SPECIAL LINEAR CHARACTERS OF FREE GROUPS OF RANK $n \geqq 4$
}

\author{
ALICE WHITTEMORE ${ }^{1}$
}

\begin{abstract}
Let $F_{n}$ be a free group of rank $n$. In a recent paper R. Horowitz has shown that for $n \leqq 3$ the ideal $I_{n}$ in the ring of special linear characters of $F_{n}$ consisting of those polynomials in the characters which vanish for all representations of $F_{n}$ by subgroups of $S L(2, C)$ is principal. In this paper the case $n=4$ is investigated; it is shown that for $n>3, I_{n}$ is not principal.
\end{abstract}

1. Introduction. The theory of two dimensional special linear characters was investigated extensively in the late nineteenth century by Fricke. More recently R. Horowitz [2] has studied the ring of special linear characters of free groups $F_{n}$; in particular, for $n=1,2$ he has shown that these rings are isomorphic respectively to the rings $Z[x]$ and $Z[x, y, z]$ of polynomials in one and three indeterminants with integer coefficients, and that the ring of characters of $F_{3}$ is isomorphic to the quotient ring of $Z\left[x_{1}, x_{2}, \cdots, x_{7}\right]$ modulo a principal ideal consisting of those polynomials which vanish on the character manifold. The first theorem of this paper shows that the number of algebraic relations between the characters of $F_{n}$ increases considerably with $n$; Theorem 2 gives some restriction on these relations for $n=4$.

2. Preliminaries; statement of theorems. Given a group $G$, denote by $(G, K)$ the set of all representations $\rho: G \rightarrow S L(2, K)$, where $K$ is either the field $\boldsymbol{R}$ or $\boldsymbol{C}$ of real or complex numbers, and where $S L(2, K)$ is the group of all $2 \times 2$ matrices with entries in $K$ and with determinant one. For $u \in G$ the character $\sigma_{u}$ of $u$ is defined by $\sigma_{u}:(G, K) \rightarrow K$, with $\rho \sigma_{u}=\sigma(u \rho)$ for all $\rho \in(G, K)$, where $\sigma(u \rho)$ denotes the trace of $u \rho$.The set $\left\{\sigma_{u} \mid u \in G\right\}$ generates a subring $J_{G, K}$ of the ring of all functions from $(G, K)$ into $K$ with the usual addition and multiplication.

Received by the editors June 19, 1972.

AMS (MOS) subject classifications (1970). Primary 20G05; Secondary 20E05, $20 \mathrm{~F} 55$.

Key words and phrases. Special linear characters, character manifold, automorphism group of a free group.

${ }^{1}$ This work was supported by a grant from the City University of New York and was greatly facilitated by the advice and suggestions of Wilhelm Magnus.

(C) American Mathematical Society 1973 
The following formulae for $u, v, w \in G[1]$ will be needed:

$$
\begin{aligned}
\sigma_{u} & =\sigma_{u^{-1}} \\
\sigma_{u v} & =\sigma_{u} \cdot \sigma_{v}-\sigma_{u v^{-1}}, \\
\sigma_{u v w} & =\sigma_{u} \cdot \sigma_{v w}+\sigma_{v} \cdot \sigma_{u w}+\sigma_{w} \cdot \sigma_{u v}-\sigma_{u} \cdot \sigma_{v} \cdot \sigma_{w}-\sigma_{u w v} .
\end{aligned}
$$

Using (2.1)-(2.3) Horowitz has proved the following theorem [2]:

Let $F_{n}$ denote the free group on $n$ free generators $a_{k}, k=1, \cdots, n$. For all $u \in F_{n}, \sigma_{u}$ can be expressed as a polynomial with integer coefficients in the $2^{n}-1$ characters

$$
\sigma_{a}, \quad a=a_{i_{1}} a_{i_{2}} \cdots a_{i_{r}}, 1 \leqq r \leqq n, 1 \leqq i_{1}<\cdots<i_{r} \leqq n .
$$

As an immediate consequence we have $J_{F_{n}, K} \cong Z_{n} / I_{n, K}$, where $Z_{n}$ is the ring of polynomials with integer coefficients in the $2^{n-1}$ indeterminants $i_{1} i_{2} \cdots i_{r}, 1 \leqq r \leqq n, 1 \leqq i_{1}<\cdots<i_{r} \leqq n$, and $I_{n, K}$ is the ideal consisting of those polynomials in $\boldsymbol{Z}_{n}$ whose images in $J_{n, K}$ under the canonical homomorphism vanish for all $\rho \in\left(F_{n}, K\right)$. It is easily checked that $I_{n, C} \subseteq I_{n, R}$, and $m \leqq n$ implies $I_{m, K} \subseteq I_{n, K}$.

Each element $\varphi$ of the group $A_{n}$ of automorphisms of $F_{n}$ induces a map $\sigma_{a} \rightarrow \sigma_{a \varphi}$ of the characters $\sigma_{a}$ of (2.4), where according to Horowitz's theorem, $\sigma_{a \varphi}$ is a polynomial $p\left(\sigma_{a}\right)$ in the $\sigma_{a}$. The corresponding assignment $i_{1} i_{2} \cdots i_{r} \rightarrow p\left(i_{1} i_{2} \cdots i_{r}\right)$ induces an automorphism of $Z_{n}$, called the automorphism induced by $\varphi$. Clearly $I_{\dot{n}, K}$ is invariant under the group $\mathscr{A}_{n}$ of automorphisms of $Z_{n}$ induced by $A_{n}$. A set of generators for $A_{n}$ is given by $A, B, C, D$, where the image of a generator $a_{k}$ of $F_{n}$ under each of these automorphisms is given by Table $1[3$, p. 164]:

\begin{tabular}{c|llllll}
\multicolumn{8}{c}{ TABLE } & & & \\
\multicolumn{1}{c}{} & $a_{1}$ & $a_{2}$ & $a_{3}$ & $\cdots$ & $a_{n-1}$ & $a_{n}$ \\
\cline { 2 - 7 } & $a_{2}$ & $a_{3}$ & $a_{4}$ & $\cdots$ & $a_{n}$ & $a_{1}$ \\
$B$ & $a_{2}$ & $a_{1}$ & $a_{3}$ & $\cdots$ & $a_{n-1}$ & $a_{n}$ \\
$C$ & $a_{1}^{-1}$ & $a_{2}$ & $a_{3}$ & $\cdots$ & $a_{n-1}$ & $a_{n}$ \\
$D$ & $a_{1} a_{2}$ & $a_{2}$ & $a_{3}$ & $\cdots$ & $a_{n-1}$ & $a_{n}$
\end{tabular}

Let $\alpha, \beta, \gamma, \delta$ denote the automorphisms of $\mathscr{A}_{n}$ induced by $A, B, C, D$ respectively. Finally, if $w=a_{j_{1}}^{ \pm 1} a_{j_{2}}^{ \pm 1} \cdots a_{j_{k}}^{ \pm 1}$ is any nonempty word in the free generators $a_{1}, \cdots, a_{n}$ of $F_{n}$, denote $\sigma_{w}$ by $\sigma_{ \pm j_{1} \pm j_{2}} \cdots{ }_{ \pm j_{k}}$. If $1 \leqq i<j<$ $k \leqq n$ by (2.3) $\sigma_{i k j}=\sigma_{i} \cdot \sigma_{j k}+\sigma_{j} \cdot \sigma_{i k}+\sigma_{k} \cdot \sigma_{i j}-\sigma_{i} \cdot \sigma_{j} \cdot \sigma_{k}-\sigma_{i j k}$; let the symbol $i k j$ represent the polynomial $i \cdot j k+j \cdot i k+k \cdot i j-i \cdot j \cdot k-i j k$ in 
1973

SPECIAL LINEAR CHARACTERS OF FREE GROUPS

385

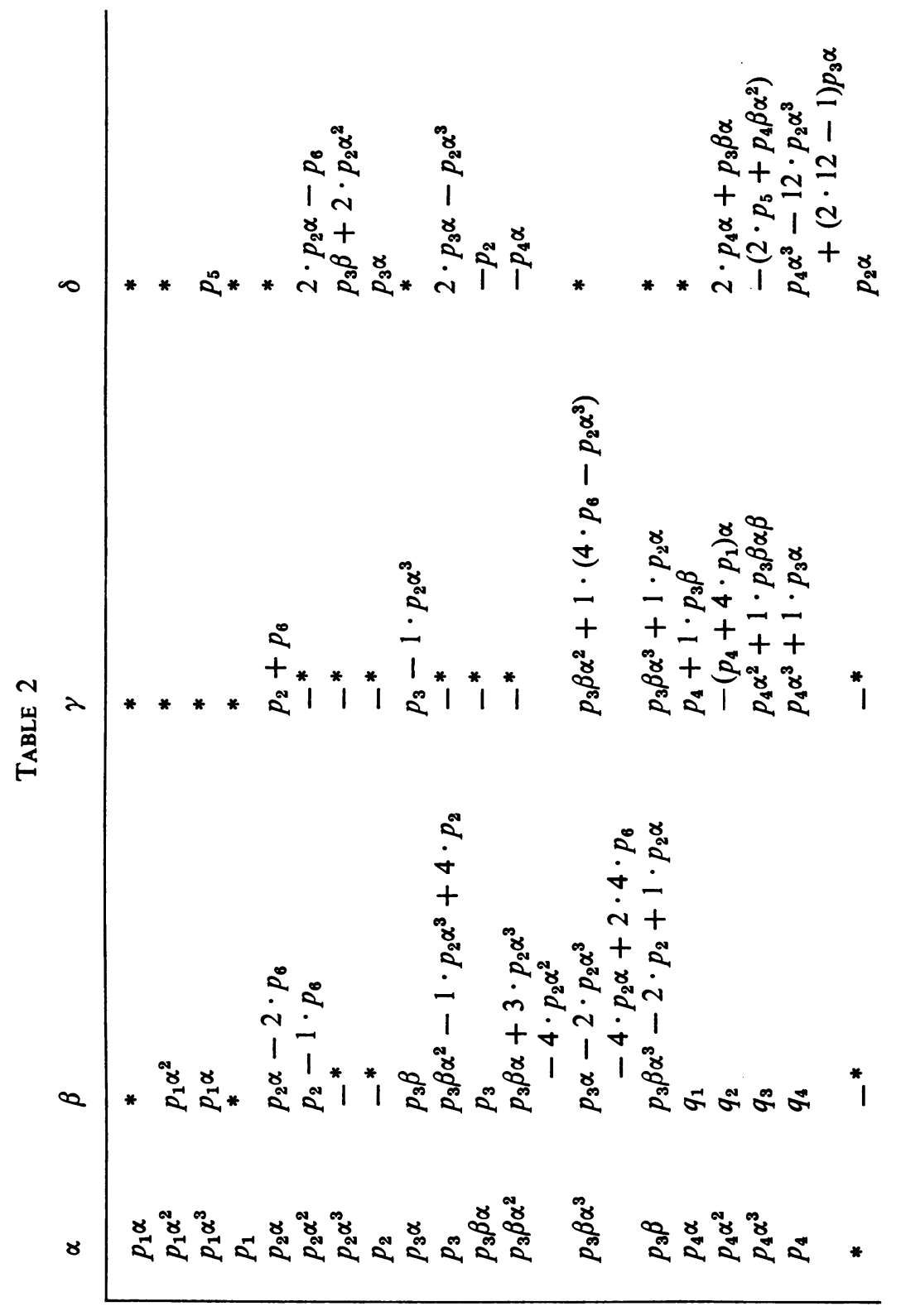

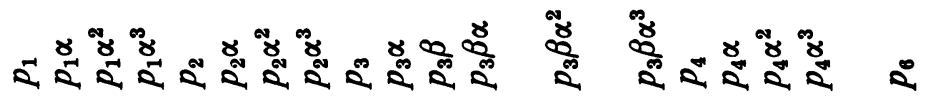


$Z_{n}$. We can now state:

THEOREM 1. In $Z_{4}$, the set of polynomials

$$
\begin{aligned}
p_{1}= & -123 \cdot 132+1^{2}+2^{2}+3^{2}+(12)^{2}+(13)^{2}+(23)^{2} \\
& -1 \cdot 2 \cdot 12-1 \cdot 3 \cdot 13-2 \cdot 3 \cdot 23+12 \cdot 13 \cdot 23-4, \\
p_{2}= & 1 \cdot 1234+243-234-12 \cdot 134+13 \cdot 124-14 \cdot 123 \\
& -1 \cdot 3 \cdot 124+3 \cdot 12 \cdot 14 \\
p_{3}= & 13 \cdot 1234-123 \cdot 134-1 \cdot 124+12 \cdot 14-2 \cdot 4+2(24) \\
& -3 \cdot 234+23 \cdot 34, \\
p_{4}= & 132 \cdot 1234-12 \cdot 23 \cdot 134-23 \cdot 234+1 \cdot 3 \cdot 134-13 \cdot 134 \\
& -12 \cdot 124+2 \cdot 23 \cdot 34+2 \cdot 12 \cdot 14+2 \cdot 24-1 \cdot 14 \\
& -3 \cdot 34+2(4), \\
p_{5}= & (1234)^{2}-1234(4 \cdot 123+3 \cdot 124+12 \cdot 34-3 \cdot 4 \cdot 12) \\
& +(123)^{2}+(124)^{2}+(12)^{2}+(34)^{2}+3^{2}+4^{2}-4 \cdot 12 \cdot 124 \\
& -3 \cdot 12 \cdot 123-3 \cdot 4 \cdot 34+34 \cdot 123 \cdot 124-4, \\
p_{6}= & 2(1234)+(1 \cdot 2-12) 34+(1 \cdot 4-14) 23+(13-1 \cdot 3) 24 \\
& -1 \cdot 234-2 \cdot 134-4 \cdot 123+3 \cdot 142
\end{aligned}
$$

together with their images

$$
\left\{p_{j} \alpha^{i} \mid i=1,2,3, j=1,2,4\right\} \cup\left\{p_{3} \alpha\right\} \cup\left\{p_{3} \beta \alpha^{i} \mid i=0,1,2,3\right\}
$$

generate an ideal I which is (i) invariant under $\mathscr{A}_{4}$; (ii) contained in $I_{4, K}$.

Theorem 1 contrasts sharply with Horowitz's results that $I_{1, K}=I_{2, K}=$ $\{0\}$, and $I_{3}, K$ is the principal ideal generated by $p_{1}$. Since $I_{m, K} \subseteq I_{n, K}$ for $m \leqq n$, the above result indicates the complexity of the ideals $I_{n, K}$ for $n \geqq 4$. On the other hand, the relations in $J_{F_{4}, K}$ are restricted by

THEOREM 2. Let $p \in I_{4, R}$ be a polynomial of degree 0 in the indeterminants $i_{1} i_{2} \cdots i_{r}$ of length $r>2$ and of nonzero degree in at most nine of the ten indeterminants of length $r \leqq 2$. Then $p \equiv 0$.

3. Proof of Theorem 1. Table 2 showing the images of the generating polynomials for $I$ under the automorphisms $\alpha, \beta, \delta, \gamma$ can be verified by direct calculation using (2.1)-(2.3) and the defining relations for $A_{4}\left[3\right.$, p. 164]. The symbols $\pm^{*}$ indicate that a given polynomial is either fixed or sent to its negative by a given automorphism. The images of $p_{5}$ and the polynomials $q_{i}(i=1,2,3,4)$ of Table 2 because of their length, are written separately. 


$$
\begin{aligned}
p_{5} \alpha= & p_{5}+1 \cdot p_{4} \alpha-3 \cdot p_{4} \alpha^{3}-34 \cdot p_{3} \beta \alpha^{3}-1 \cdot 34 p_{2} \alpha \\
& +14 \cdot\left(p_{3} \beta+2 \cdot p_{2} \alpha^{2}\right), \\
p_{5} \beta= & p_{5}+\left[1 \cdot\left(1 \cdot p_{1} \alpha+p_{4} \alpha+p_{4} \beta \alpha\right)+4 \cdot\left(4 \cdot p_{1}+p_{4}+p_{4} \alpha^{3} \beta \alpha\right)\right] \alpha \\
& +1 \cdot 2 \cdot\left(p_{3} \beta \alpha+p_{3} \beta \alpha \beta+34 \cdot p_{6}\right), \\
p_{5} \gamma= & p_{5}+1 \cdot\left[\left(4 \cdot p_{1}+p_{4}+p_{4} \alpha^{3} \beta \alpha\right) \alpha\right], \\
p_{5} \delta= & 2^{2} \cdot p_{5}+\left(4 \cdot p_{4} \beta+4 \cdot p_{4}+p_{1}\right) \alpha^{2}, \\
q_{1}= & p_{4}+(1 \cdot 2-12) p_{2} \alpha^{2}-2 p_{3}+1 \cdot p_{3} \beta, \\
q_{2}= & 1 \cdot 4 \cdot p_{2} \alpha^{2}+3 \cdot p_{3} \alpha \beta \\
& -\left(p_{4} \beta \alpha^{2}+2 \cdot p_{1} \alpha^{2}+4 \cdot p_{3}+1 \cdot p_{3} \beta \alpha+1 \cdot 34 \cdot p_{6}\right), \\
q_{3}= & {\left[23 \cdot p_{2}+(1 \cdot 2-12) p_{2} \alpha^{2}-p_{4} \beta-4 \cdot p_{1}-2 \cdot p_{3}\right] \alpha-2 \cdot 34 \cdot p_{6}, } \\
q_{4}= & p_{4} \alpha^{3}+2 \cdot p_{3} \beta \alpha^{2}+2 \cdot 4 \cdot p_{2}-1 \cdot p_{3} \alpha-12 \cdot p_{2} \alpha^{3} .
\end{aligned}
$$

It is evident from the table that $I$ is invariant under $\mathscr{A}_{4}$. In order to show $I \subseteq I_{4, K}$, because of the relations $p_{2}=-p_{3} \beta \delta, \quad p_{4}=p_{3} \beta \alpha \delta \alpha^{-1}$, $p_{5}=-\left(p_{4} \alpha^{2} \delta+p_{4} \beta \alpha^{2}\right), p_{1}=p_{5}\left(\alpha_{1}^{2} \delta\right)^{-1}, p_{6}=p_{2} \alpha \delta^{-1}$ and the fact that $I_{4, K}$ is invariant under $\mathscr{A}_{4}$, one need only show $p_{3} \in I_{4, K}$. For this purpose let $u=a_{1} a_{2} a_{3}, v=a_{1} a_{3} a_{4}$. By (2.2) we have

$$
0=-\sigma_{123} \cdot \sigma_{134}+\sigma_{123134}+\sigma_{23-3-4} \text {. }
$$

Rewriting $\sigma_{23-3-4}$ by (2.1) and (2.2) one obtains

$$
\sigma_{23-3-4}=\sigma_{23} \sigma_{34}-\sigma_{3} \cdot \sigma_{234}+\sigma_{24} \text {. }
$$

Expansion of $\sigma_{123134}$ via (2.3) with $u=a_{1} a_{2}, v=a_{3} a_{1} a_{3}, w=a_{4}$ using (2.1), (2.2) yields

$$
\sigma_{123134}=\sigma_{13} \cdot \sigma_{1234}-\sigma_{1} \cdot \sigma_{124}+\sigma_{12} \cdot \sigma_{14}-\sigma_{2} \cdot \sigma_{4}+\sigma_{24} .
$$

Substitution of (3.2), (3.3) into (3.1) yields the desired result.

4. Proof of Theorem 2. We may assume without loss of generality that $p$ has degree 0 in either of the indeterminants 4 or 34 . Indeed, if $p$ has degree 0 in one of the remaining eight indeterminants of length $k \leqq 2, p$ may be replaced by $p \alpha^{\lambda} \beta^{\varepsilon}$ for suitable $\lambda=0,1,2,3, \varepsilon=0,1$. Both cases are treated simultaneously.

Define a representation $\rho: F_{4} \rightarrow S L(2, R)$ as follows:

$$
\begin{gathered}
a_{1} \rho=\left[\begin{array}{cc}
\lambda_{1} & 0 \\
0 & \lambda_{1}^{-1}
\end{array}\right], \quad a_{2} \rho=\left[\begin{array}{cc}
\lambda_{2} & \lambda_{2} \lambda_{3}-1 \\
1 & \lambda_{3}
\end{array}\right], \quad a_{3} \rho=\left[\begin{array}{cc}
\lambda_{4} & \lambda_{5} \\
\lambda_{6} & \frac{\lambda_{5} \lambda_{6}+1}{\lambda_{4}}
\end{array}\right], \\
a_{4} \rho=\left[\begin{array}{cc}
\lambda_{7} & \lambda_{8} \\
\lambda_{9} & \frac{\lambda_{8} \lambda_{9}+1}{\lambda_{7}}
\end{array}\right], \quad \lambda=\left(\lambda_{1}, \cdots, \lambda_{9}\right) \in R_{9}, \lambda_{1}, \lambda_{4}, \lambda_{7} \text { nonzero. }
\end{gathered}
$$


The two systems of functional equations $\lambda\left(\rho \sigma_{i}\right)=\xi_{i}, i=1,2,3,12,13$, $14,23,24, k, k=4$ and $k=34$, define transformations $F_{1}$ and $F_{2}$ respectively from a subregion of $\boldsymbol{R}_{9}$ into $\boldsymbol{R}_{9}$. Let $\bar{\lambda} \in \boldsymbol{R}_{9}$ have coordinates $\lambda_{1}=2, \lambda_{i}=1$, $1<i \leqq 9$. The Jacobeans of both $F_{1}$ and $F_{2}$ evaluated at $\bar{\lambda}$ are nonzero. It follows from the implicit function theorem that there exist neighborhoods $U_{i}$ of $\bar{\lambda} F_{i}$ and transformations $G_{i}: U_{i} \rightarrow \boldsymbol{R}_{\mathbf{9}}(i=1,2)$ with the property that $\xi\left(G_{i} \circ F_{i}\right)=\xi$ for all $\xi \in U_{i}$. Accordingly, for all $\xi \in U_{i}$ there exist representations $\rho_{i} \in\left(F_{4}, R\right)(i=1,2)$ such that $\xi=\left(\rho_{1} \sigma_{1}, \cdots\right.$, $\left.\rho_{1} \sigma_{24}, \rho_{1} \sigma_{4}\right), i=1 ; \xi=\left(\rho_{2} \sigma_{1}, \cdots, \rho_{2} \sigma_{24}, \rho_{2} \sigma_{34}\right), i=2$.

Since $p \in I_{4, R}$ we have $p(\xi)=0$ for all $\xi \in U_{i}$, which implies $p \equiv 0$.

\section{REFERENCES}

1. R. Fricke and F. Klein, Vorlesungen über die Theorie der automorphen Functionen. Vol. 1, Teubner, Leipzig, 1897.

2. R. Horowitz, Characters of free groups represented in the two-dimensional special linear group, Comm. Pure Appl. Math. 25 (1972), 635-650.

3. W. Magnus, A. Karass and D. Solitar, Combinatorial group theory: Presentations of groups in terms of generators and relations, Pure and Appl. Math., vol. 13, Interscience, New York, 1966. MR 34 \#7617.

Department of Mathematics, Hunter College (CUNy), New York, New YORK 10021 